\title{
CORRIGENDUM
}

\section{Assessment of the data quality for the NHEXAS - Part II: Minnesota children's pesticide exposure study (MNCPES)}

\author{
EDO D. PELLIZZARI, DORIS J. SMITH, C. ANDREW CLAYTON AND JAMES J. QUACKENBOSS
}

Journal of Exposure Analysis and Environmental Epidemiology (2004) 14, 108. doi:10.1038/sj.jea.7500333

Journal of Exposure Analysis and Environmental Epidemiology (2003) 13, 465-479. doi:10.1038/sj.jea. 7500315
Since the publication of the above paper, the authors have identified an error in Table 14. A revised version of the table is given below.

Table 14. Detection limits and percent measurables for PAHs in air, dust wipe, and food samples

\begin{tabular}{|c|c|c|c|c|c|c|c|c|c|c|c|}
\hline \multirow[t]{3}{*}{ PAH } & \multicolumn{5}{|l|}{ Air } & \multicolumn{3}{|c|}{ Wipe } & \multicolumn{3}{|c|}{ Food } \\
\hline & \multirow[t]{2}{*}{$N^{\mathrm{a}}$} & \multirow{2}{*}{$\begin{array}{l}\operatorname{Median}^{\mathrm{b}} \\
\operatorname{MDL}\left(\mu \mathrm{g} / \mathrm{m}^{3}\right)\end{array}$} & \multicolumn{3}{|c|}{ Percent measurable } & \multirow[t]{2}{*}{$N$} & \multirow{2}{*}{$\begin{array}{l}\text { Median }^{\mathrm{d}} \\
\text { QL }\left(\mu \mathrm{g} / \mathrm{cm}^{2}\right)\end{array}$} & \multirow{2}{*}{$\begin{array}{l}\text { Percent } \\
\text { measurable }\end{array}$} & \multirow[t]{2}{*}{$N$} & \multirow{2}{*}{$\begin{array}{l}\text { Median }^{\mathrm{d}} \\
\mathrm{QL}(\mu \mathrm{g} / \mathrm{kg})\end{array}$} & \multirow{2}{*}{$\begin{array}{l}\text { Percent } \\
\text { measurable }\end{array}$} \\
\hline & & & $\mathrm{P}^{\mathrm{c}}$ & $\mathrm{I}^{\mathrm{c}}$ & $\mathrm{O}^{\mathrm{c}}$ & & & & & & \\
\hline Benzo(a)pyrene ${ }^{e}$ & 218 & 0.01 & 58 & 43 & 48 & 137 & 0.21 & 19 & 67 & 0.01 & 69 \\
\hline Benzo(a)anthracene $e^{\mathrm{e}}$ & 231 & 0.04 & 75 & 35 & 42 & 207 & 0.21 & 21 & 70 & 0.06 & 71 \\
\hline Acenaphthylene & 84 & 0.02 & 81 & 68 & 95 & 206 & 0.21 & 2.9 & 66 & 0.07 & 67 \\
\hline Anthracene & 228 & 0.20 & 100 & 96 & 61 & 207 & 0.21 & 22 & 69 & 0.02 & 71 \\
\hline Chrysene & 233 & 0.01 & 93 & 84 & 63 & 207 & 0.21 & 27 & 70 & 0.009 & 71 \\
\hline Benzo(e)pyrene & 230 & 0.04 & 70 & 38 & 48 & 204 & 0.21 & 5.9 & 56 & 0.02 & 58 \\
\hline Benzo(ghi)perylene & 234 & 0.01 & 94 & 72 & 63 & 207 & 0.21 & 15 & 75 & 0.02 & 76 \\
\hline Benzo(k)fluoranthene & 220 & 0.008 & 59 & 29 & 29 & 206 & 0.21 & 22 & 67 & 0.03 & 67 \\
\hline Fluoranthene & 233 & 0.07 & 100 & 100 & 100 & 200 & 0.21 & 24 & 74 & 0.25 & 72 \\
\hline Pyrene & 233 & 0.08 & 100 & 100 & 100 & 206 & 0.21 & 19 & 72 & 0.03 & 73 \\
\hline Indeno[1,2,3-cd]pyrene & 233 & 0.11 & 44 & 8.2 & 14 & 207 & 0.21 & 17 & 75 & 0.004 & 76 \\
\hline Benzo(b)fluoranthrene & 229 & 0.02 & 94 & 81 & 71 & 206 & 0.21 & 7.8 & 69 & 0.05 & 70 \\
\hline Phenanthrene & 234 & 0.43 & 100 & 100 & 98 & 202 & 0.21 & 47 & 75 & 0.11 & 76 \\
\hline
\end{tabular}

${ }^{\mathrm{a}}$ Number of observations for median.

${ }^{\mathrm{b}}$ Method detection limit.

${ }^{\mathrm{c}} \mathrm{P}=$ personal, $\mathrm{I}=$ indoor, $\mathrm{O}=$ outdoor .

${ }^{\mathrm{d}}$ Quantification limit.

${ }^{\text {e}}$ Primary target PAHs for NHEXAS/MNCPES. 